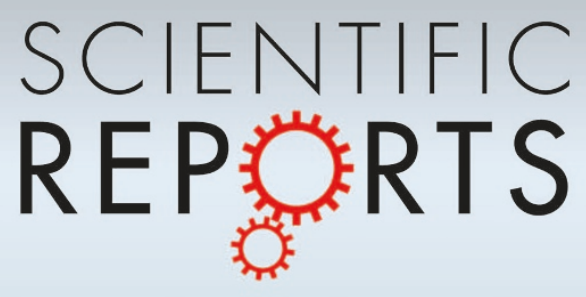

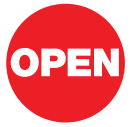

SUBJECT AREAS: ELECTRONIC PROPERTIES AND DEVICES

TWO-DIMENSIONAL MATERIALS

ELECTRICAL AND ELECTRONIC ENGINEERING

ELECTRONIC DEVICES

Received

8 June 2012

Accepted

10 January 2013

Published

13 February 2013

Correspondence and requests for materials should be addressed to

X.D. (xduan@chem. ucla.edu)

* Current address: Department of Electronic and

Electrical Engineering, Sungkyunkwan University, Suwon 440-746, South Korea.

\title{
Tunable transport gap in narrow bilayer graphene nanoribbons
}

\author{
Woo Jong $\mathrm{Yu}^{1 *}$ \& Xiangfeng Duan ${ }^{1,2}$
}

\author{
'Department of Chemistry and Biochemistry, University of California, Los Angeles, CA 90095, USA, ${ }^{2}$ California Nanosystems \\ Institute, University of California, Los Angeles, CA 90095, USA.
}

The lack of a bandgap makes bulk graphene unsuitable for room temperature transistors with a sufficient on/off current ratio. Lateral constriction of charge carriers in graphene nanostructures or vertical inversion symmetry breaking in bilayer graphene are two potential strategies to mitigate this challenge, but each alone is insufficient to consistently achieve a large enough on/off ratio (e.g. $>1000)$ for typical logic applications. Herein we report the combination of lateral carrier constriction and vertical inversion symmetry breaking in bilayer graphene nanoribbons (GNRs) to tune their transport gaps and improve the on/off ratio. Our studies demonstrate that the on/off current ratio of bilayer GNRs can be systematically increased upon applying a vertical electric field, to achieve a largest on/off current ratio over 3000 at room temperature.

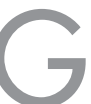

raphene has attracted considerable interest as a new electronic material for both fundamental investigations and potential applications due to its unique electronic properties including the highest room temperature carrier mobility and carrier saturation velocity of all known materials ${ }^{1-5}$. However, the bulk graphene sheet is a semimetal with a zero bandgap and cannot be used for room temperature transistors with sufficient on/off ratio, which remains a main challenge to apply graphene for digital electronic or photonic applications $^{6,7}$. A number of approaches have been proposed for inducing a bandgap in mono- or bilayer graphene. A first strategy is to create graphene nanostructures with lateral constriction and confinement of charge carriers ${ }^{8-19}$. In particular, graphene nanoribbons (GNRs) of sub-10 nm width are predicted to be semiconducting due to lateral confinement of the electron wave function in the transverse direction and edge disorder induced Anderson localization. Both theoretical and experimental studies have demonstrated that Anderson localization along with lateral confinement can be achieved in GNRs ${ }^{8-18}$, nanomeshes ${ }^{19}$, or quantum dots ${ }^{20}$ to open up a transport gap inversely proportional to the conducting channel width. An on/off ratio up to 100 is typically achieved at room temperature in these graphene nanostructures with occasionally larger values achieved in ultra-small device ${ }^{17,19,20}$. An alternative strategy is to break the inversion symmetry in bilayer graphene $e^{21-25}$. By applying external electric field normal to the bilayer graphene plane, band gap can be opened in the Bernalstacking (AB-stacking) bilayer graphene. In particular, bilayer graphene field effect transistors (FETs) with on/off ratio up to 100 have been achieved at the room temperature ${ }^{24}$.

The combination of both methods together has the potential to open up a larger bandgap in bilayer GNRs. The theoretical studies have suggested that a vertical field could be used to further modulate the band gap in a bilayer or tri-layer GNRs ${ }^{26-29}$, but there is yet a lack of experimental investigation on this topic to date. Herein, we report the first experimental demonstration that a combination of lateral carrier constriction and vertical inversion symmetry breaking in bilayer GNRs can be used to achieve graphene devices with further improved on/off ratios. We show that the on/off current ratio of bilayer GNRs can be increased greatly to reach up to 3000 at room temperature by applying a vertical electric field. This on/off current ratio of our bilayer GNR FET is 30 fold higher than the highest value $(\sim 100)$ reported previously for bilayer graphene devices ${ }^{24}$.

\section{Results}

Bilayer GNRs were produced by employing thin nanowires as a physical protection mask in oxygen plasma etching process. Figure 1 illustrates the fabrication steps to obtain dual gated bilayer GNR FETs. Bilayer graphene on the silicon substrate was prepared as a starting material through exfoliation from natural graphite and physical dry transfer process, although the approach described here can be readily extended to graphene sheets or films obtained through chemical approaches ${ }^{29-32}$. The layers of graphene were classified based on their optical contrast and Raman spectroscopic studies (Fig. S1) ${ }^{33,34}$. The $\mathrm{Si} / \mathrm{Al}_{2} \mathrm{O}_{3}$ core/shell nanowires were then aligned on top of bilayer graphene through a physical dry transfer process (Fig. 1a) ${ }^{35,36}$, followed by the definition of source and 
(a)

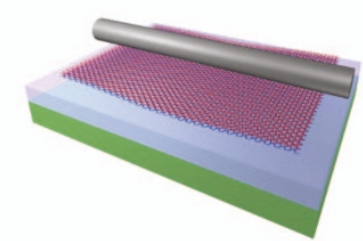

$\mathrm{Si}-\mathrm{Al}_{2} \mathrm{O}_{3}$ coreshell nanowire on bilayer graphene

(d)

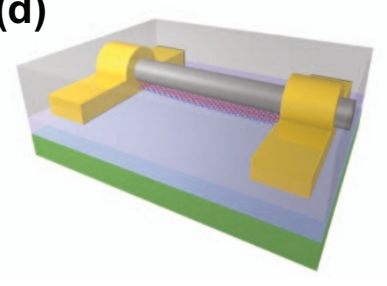

Deposition of $\mathrm{HfO}_{2}$ as a protecting layer (b)

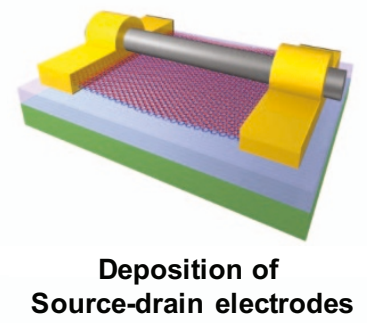

(e)

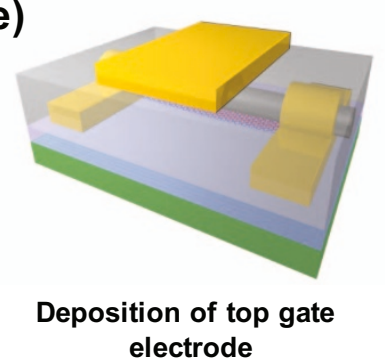

(c)

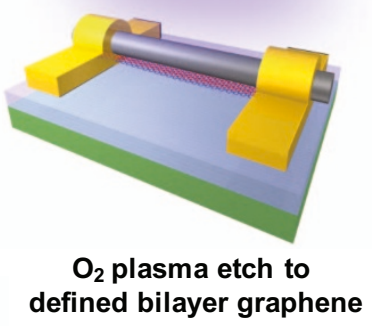

(f)

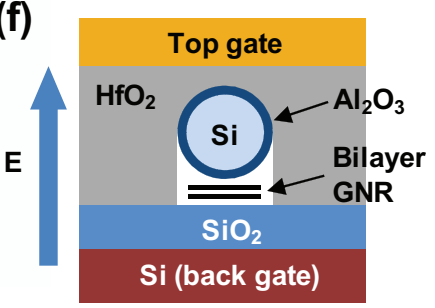

Figure 1 Schematic illustration of the fabrication procedures to obtain dual-gated bilayer graphene nanoribbon $\mathrm{FET}$. (a), $\mathrm{Si} / \mathrm{Al}_{2} \mathrm{O}_{3}$ core/shell nanowires are transferred onto a bilayer graphene sheet. (b), Source and drain electrodes are formed on aligned bilayer graphene and Si/Al $\mathrm{O}_{3}$ core/shell nanowires. (c), Uncovered bilayer graphene is etched by oxygen plasma, and bilayer graphene nanoribbons are produced underneath Si nanowires. (d), A thin film of $60 \mathrm{~nm} \mathrm{HfO}_{2}$ is deposited by e-beam evaporation to prevent electrical breakdown of plasma damaged $\mathrm{SiO}_{2}$. (e), Top gate electrode is deposited as a final process. (f), Schematic of the cross-sectional view of the device.

drain electrodes (Ti/Au: $30 \mathrm{~nm} / 60 \mathrm{~nm}$ ) using e-beam lithography and e-beam evaporation (Fig. $1 \mathrm{~b}$ ). The $\mathrm{Si} / \mathrm{Al}_{2} \mathrm{O}_{3}$ core/shell nanowires were produced by deposition of $2 \mathrm{~nm} \mathrm{Al}_{2} \mathrm{O}_{3}$ on the Si nanowire surface using an atomic layer deposition (ALD) process. TEM studies show a uniformly coated $\mathrm{Al}_{2} \mathrm{O}_{3}$ layer on $\mathrm{Si}$ nanowire surface (Fig. $\mathrm{S} 2 \mathrm{a}, \mathrm{b})$. The $\mathrm{Si} / \mathrm{Al}_{2} \mathrm{O}_{3}$ nanowire mask is not removed from the device, and the resistance of the nanowire itself is typically larger than 1 teraohm and would not affect the charge transport of the final devices (Fig. S2c, d). Oxygen plasma was used to remove the exposed graphene, leaving only the graphene protected underneath the $\mathrm{Si} / \mathrm{Al}_{2} \mathrm{O}_{3}$ nanowire to form a GNR connected to two big blocks of graphene protected under the source and drain electrodes (Fig. 1c) ${ }^{17}$. A $60 \mathrm{~nm}$ $\mathrm{HfO}_{2}$ protecting layer was then deposited on the top of device using e-beam evaporation to prevent electrical breakdown of the oxygen plasma damaged $\mathrm{SiO}_{2}$ back gate dielectric under high field (Fig. 1d). It was found that $\mathrm{SiO}_{2}$ back gate dielectric (after exposure to oxygen plasma) can be easily broken down by the relatively high vertical electric field (e.g. at $\mathrm{V}_{\mathrm{BG}} \sim 50 \mathrm{~V}$ ). The typical breakdown voltage was increased to $\mathrm{V}_{\mathrm{BG}} \sim 120 \mathrm{~V}$ with the deposition of the $\mathrm{HfO}_{2}$ protecting layer. Lastly, top gate electrode of (Ti/Au: $30 \mathrm{~nm} / 60 \mathrm{~nm}$ ) was fabricated using conventional e-beam lithography (Fig. 1e). Figure if shows cross sectional view of dual-gated bilayer GNR FET. In this device, heavily $\mathrm{n}$-doped silicon wafer was used as a back gate and $300 \mathrm{~nm}$ of $\mathrm{SiO}_{2}$ on the silicon wafer was used as back gate dielectric. Bilayer GNR is located underneath of $\mathrm{Si}-\mathrm{Al}_{2} \mathrm{O}_{3}$ core-shell nanowire, and channel width of bilayer GNR is defined by nanowire diameter. Top gate dielectric consists of $\mathrm{Si} / \mathrm{Al}_{2} \mathrm{O}_{3}$ core/shell nanowire and ebeam evaporation deposited $\mathrm{HfO}_{2}$. Top gate electrode is located on the top dielectric. Vertical electric field is applied between top and back gates to break the symmetry of bilayer GNR. Optical images of each fabrication steps are shown in Figure S3.

Channel narrowing was used as a first step to open up a transport gap in bilayer graphene (Fig. 2). Large area bilayer graphene is a semimetal with zero bandgap (Fig. 2a). A transport gap can be opened by etching large area graphene into one dimensional graphene nanoribbons due to the lateral confinement of the electron wave function in the transverse direction and edge disorder induced Anderson localization (Fig. 2b) ${ }^{8-19}$. Our previous studies have shown that the initial etching is predominantly a vertical etch and can result in GNRs with width comparable to the nanowire diameter ${ }^{17}$. In our bilayer graphene FET, channel width was reduced from $\sim 2.5 \mu \mathrm{m}$ to $\sim 40 \mathrm{~nm}$ of nanowire diameter after 60 s plasma etching. At the same time, on-current was significantly reduced from $\sim 85 \mu \mathrm{A}$ to $\sim 250 \mathrm{nA}$ and off-current was decreased from $60 \mu \mathrm{A}$ to $100 \mathrm{nA}$, with the corresponding on/off current ratio improved from 1.4 to $\sim 2.5$ (Fig. 2c). The width of the GNRs can be further scaled down with additional etching steps that undercut the graphene underneath the nanowire through lateral etching ${ }^{17}$. The transfer characteristics of bilayer graphene nanoribbon show that the on/off current ratio of bilayer GNR FET was gradually improved from $\sim 2.5$ to $\sim 100$ with increasing etching time from $60 \mathrm{~s}$ to $240 \mathrm{~s}$ in steps of $60 \mathrm{~s}$ (Fig. 2c). It should be noted that the I-V characteristics of our bilayer GNR FET is rather rough, which might be attributed to the edge disorder in narrow GNRs created by plasma etching process ${ }^{37}$. In general, electrical noise increases with narrowing channel width and increasing etching time (Fig. 2c).

To further increase the transport bandgap and improve the on/off current ratio, vertical electric field was applied across the bilayer GNR to break the inversion symmetry (Fig. 3). Figure 3a schematically illustrates the change of the band gap of bilayer graphene upon application of vertical electric field. Bilayer graphene has a gapless band structure that valence and conduction bands have a point contact at the $\mathrm{K}$ point. If an electric field is applied perpendicular to the bilayer graphene, conduction band and valence band curves upwards and downwards near the K point, respectively, and a finite bandgap is created (Fig. 3a) ${ }^{38}$. It has been theoretically predicted that conduction and valence band of bilayer GNR can be tuned in a similar way to those of bilayer graphene sheet under a vertical electric field 
(a)

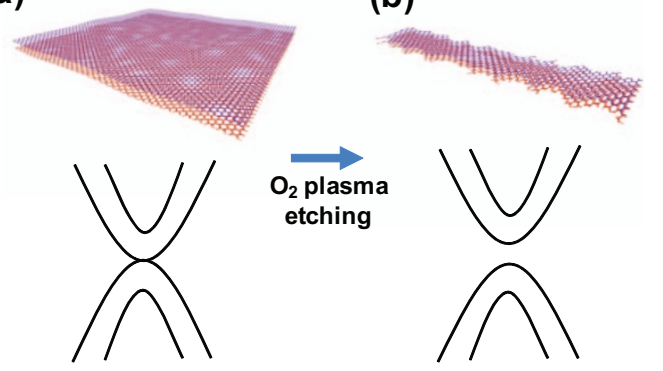

(c)

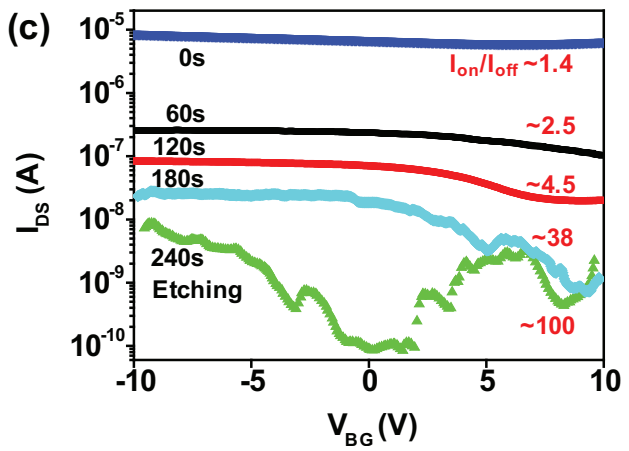

Figure $2 \mid$ The opening of transport gap in bilayer graphene by narrowing channel width. (a-b), Schematic images of (a), bilayer graphene sheet and (b), bilayer graphene nanoribbon with corresponding band diagrams. (c), Transfer characteristics of a bilayer GNR FET at the various oxygen plasma etching time $\left(\mathrm{V}_{\mathrm{DS}}=0.1 \mathrm{~V}\right)$. The etching time was varied from $0 \mathrm{~s}$ to $240 \mathrm{~s}$ from top to bottom in steps of $60 \mathrm{~s}$.

(a)
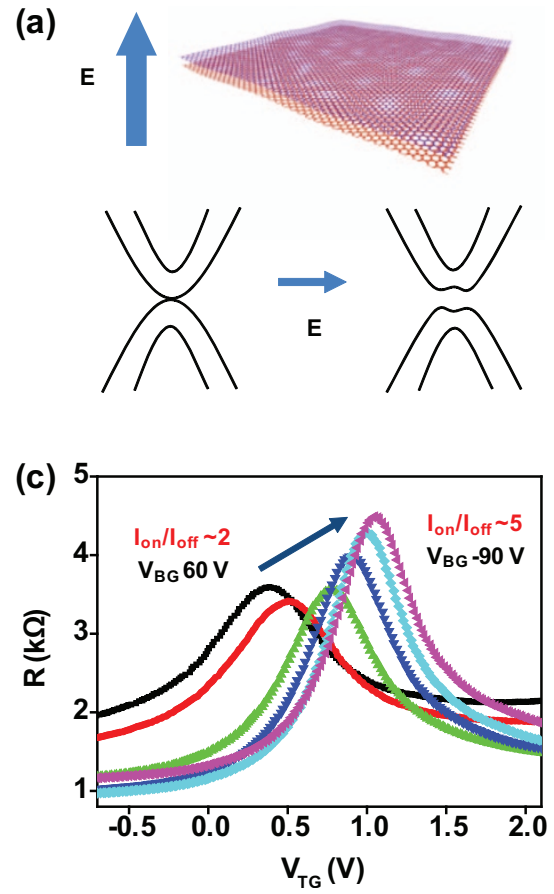

(Fig. 3b ${ }^{26,27}$. In this way, a significantly larger bandgap can be achieved in bilayer GNRs than the bulk bilayer graphene sheet under the same vertical electric field. Figure $3 \mathrm{c}$ shows the switching characteristics of a bulk bilayer graphene FET with $2.7 \mu \mathrm{m}$ wide by $4.5 \mu \mathrm{m}$ long channel. Each curves was obtained by sweeping the top gate bias $\left(\mathrm{V}_{\mathrm{TG}}\right)$ from -0.7 to $2.1 \mathrm{~V}$ with a fixed drain-source bias $\left(\mathrm{V}_{\mathrm{DS}}\right)$ of $100 \mathrm{mV}$ and variable back gate bias $\left(\mathrm{V}_{\mathrm{BG}}\right)$ from 60 to $-90 \mathrm{~V}$ at steps of $30 \mathrm{~V}$. The off-resistance increases in both directions of back gate voltage, which is consistent with previous reports on bilayer graphene devices ${ }^{24}$. The on/off ratio was improved from 2 to 5 by changing $V_{B G}$ from $60 \mathrm{~V}$ to $-90 \mathrm{~V}$. Figure $3 \mathrm{~d}$ shows the switching characteristics of a bilayer GNR FET with a $\sim 20 \mathrm{~nm}$ channel width, $\sim 1.5 \mu \mathrm{m}$ length, at a constant $\mathrm{V}_{\mathrm{DS}}$ of $100 \mathrm{mV}$ and variable $\mathrm{V}_{\mathrm{BG}}$ from 60 to $-100 \mathrm{~V}$ at steps of $20 \mathrm{~V}$. The on/off current ratio was initially $\sim 10$ which is higher than graphene sheet in Figure $3 \mathrm{c}$ because of lateral confinement and edge disorder induced Anderson localization, and improved to $\sim 70$ by changing $\mathrm{V}_{\mathrm{BG}}$ from 60 to $-100 \mathrm{~V}$ by the breaking inversion symmetry. This result is well matched with theoretical prediction of bandgap structures evolution as illustrated in Figure 3b.

The final on/off current ratio of the bilayer GNR FET upon application of the vertical electric field is highly dependent on the initial on/off ratio without vertical electric field. We have studied gap opening in bilayer GNR with an initially high on/off current ratio of $\sim 400$ (Fig. 4). Figure 4a shows the switching behaviour of the bilayer GNR FET measured at room temperature. $V_{\text {TG }}$ was scanned from $0 \mathrm{~V}$ to $6 \mathrm{~V}$ while the $\mathrm{V}_{\mathrm{BG}}$ was varied from 0 to $-90 \mathrm{~V}$ at steps of $30 \mathrm{~V}$. This bilayer GNR FET showed an expected increase in offresistance with increasing back gate voltage. Importantly, the transfer characteristics show the on/off current ratio was significantly improved from $\sim 400$ to $>3000$ at room temperature (Fig. 4b). This represents the highest room-temperature on/off current ratio reported for bilayer graphene and is 30 fold higher than the highest value $(\sim 100)$ reported previously ${ }^{24}$. Figure $4 \mathrm{c}$ shows the output characteristics of the same device at variable $\mathrm{V}_{\mathrm{TG}}$ and a fixed $\mathrm{V}_{\mathrm{BG}}$ of

(b)
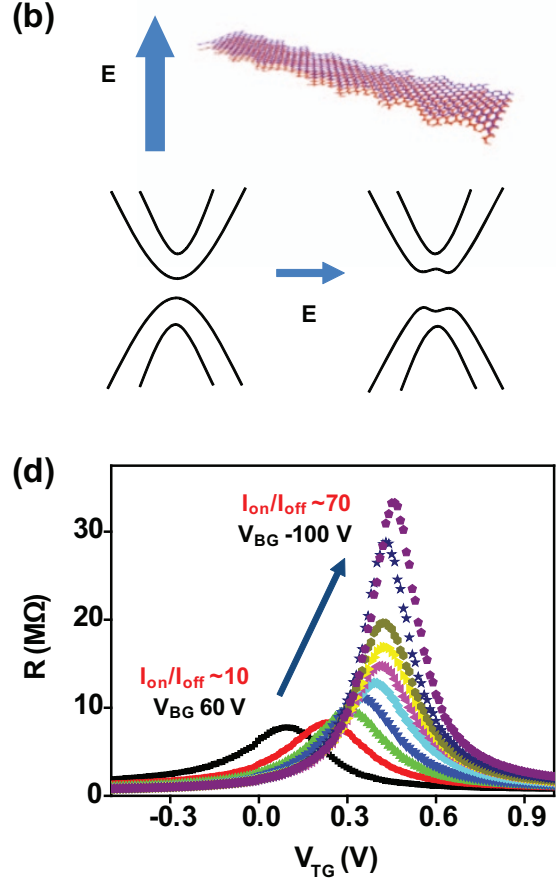

Figure 3 The further opening of transport gap in bilayer graphene nanoribbon by breaking the inversion symmetry. (a-b), Schematic images of (a), bilayer graphene sheet and (b), bilayer graphene nanoribbon with a vertical electrical displacement field and corresponding energy band structure before and after applying the displacement field. (c), Switching behavior of a bilayer graphene sheet FET at $\mathrm{V}_{\mathrm{DS}}=0.1 \mathrm{~V}$ and $\mathrm{V}_{\mathrm{BG}}$ varied from 60 to $-90 \mathrm{~V}$ in steps of $30 \mathrm{~V}$. (d), Switching behavior of a bilayer graphene nanoribbon FETs at $\mathrm{V}_{\mathrm{DS}}=0.1 \mathrm{~V}$ and $\mathrm{V}_{\mathrm{BG}}$ varied from 60 to $-100 \mathrm{~V}$ in steps of $20 \mathrm{~V}$. 

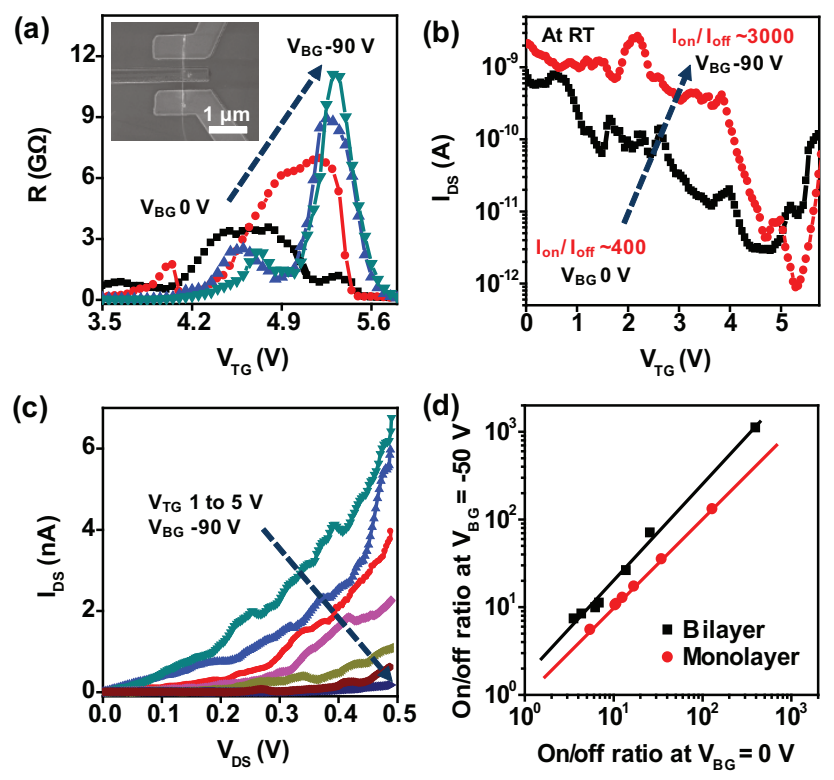

Figure $4 \mid$ High on/off ratio bilayer graphene nanoribbon FET.

(a-b), The room temperature (a), switching behavior and (b), transfer characteristics of the narrow bilayer graphene nanoribbon FET at the various back gate voltages $\left(\mathrm{V}_{\mathrm{DS}}=10 \mathrm{mV}\right)$. $\mathrm{V}_{\mathrm{BG}}$ was modulated from 0 to $-90 \mathrm{~V}$ in steps of $30 \mathrm{~V}$. Inset in (a) shows SEM image of the bilayer graphene nanoribbon FET used in this measurement. (c), $\mathrm{I}_{\mathrm{DS}}-\mathrm{V}_{\mathrm{DS}}$ output characteristics of the narrow bilayer graphene nanoribbon FET with $\mathrm{V}_{\mathrm{TG}}$ varied from 1 to $5 \mathrm{~V}$ in steps of $0.5 \mathrm{~V}\left(\mathrm{~V}_{\mathrm{BG}}=-90 \mathrm{~V}\right)$. (d), Relations between the on/off current ratio of monolayer and bilayer GNR FETs before and after application of the vertical electric field. The vertical and horizontal axes indicate that the on/off current ratio before and after the application of the vertical electric field.

$-90 \mathrm{~V}$. The transport gap of the bilayer GNR FET can be estimated to be $\sim 400 \mathrm{meV}$ at the $\mathrm{V}_{\mathrm{BG}}=-90 \mathrm{~V}$ based on the relation $I_{\text {on }} / I_{\text {off }} \approx$ $\exp \left(-q \phi_{\text {barrier }} / k_{B} T\right)$ and $E_{g}=2 q \phi_{\text {barrier }}$, assuming the schottky barrier height is about half of the electrical bandgap (Fig. S4) ${ }^{18,24,39}$. Here, $\phi_{\text {barrier }}$ is the schottky barrier height, $k_{B}$ is the Boltzmann constant, $T$ is the temperature, and $E_{g}$ is the bandgap.

We have investigated a large number of monolayer and bilayer GNR devices. The on/off current ratio of bilayer GNRs (black square) and monolayer GNRs (red circle) FETs at the $\mathrm{V}_{\mathrm{BG}}=-50 \mathrm{~V}$ are plotted against the on/off current ratio at the $\mathrm{V}_{\mathrm{BG}}=0 \mathrm{~V}$ (Fig. $4 \mathrm{~d}$ ). The plot clearly shows that the on/off current ratio of monolayer GNR FETs is not changed by application of vertical electric field (Fig. 4d, S5). On the other hand, all of the bilayer GNR FETs showed an obvious increase in on/off current ratios upon the application of a vertical electric field. The final on/off current ratio of bilayer GNR FETs are highly dependent on its initial on/off ratio.

\section{Discussion}

We have demonstrated that a larger transport gap can be induced in bilayer GNRs by combining two strategies of lateral constriction of charge carriers and vertical inversion symmetry breaking. A highest room temperature on/off current ratio over 3000 can be achieved upon applying a vertical electric field across a bilayer GNR with an initial on/off ratio of 400 . This on/off ratio greatly exceeds the typical values obtained in graphene nanostructures or bilayer graphene devices, and is already large enough for logical applications. We should also note that although a highest on/off ratio exceeding 3000 is achieved, most of devices showed low on/off ratio around 100 or smaller. Importantly, our studies demonstrate that the on/off current ratio of the bilayer GNRs can be consistently improved upon the application of a vertical electric field, and the final on/off ratio of the bilayer GNRs with the vertical field is highly dependent on the initial on/off ratio without the vertical field. The on/off ratio of the bilayer GNR devices can be further enhanced through several strategies. First, the current devices do not have an effective alignment between the source-gate-drain electrodes, with the gate only modulate $\sim 50 \%$ of the channel. A better alignment of source, drain, and gate electrodes can not only improve gate modulation of bilayer GNR device but also enhance the inversion-symmetry breaking in bilayer graphene. Secondly, the on-current of the graphene transistors can also be enhanced by reducing the graphene-metal contact resistance. The off-current can be further suppressed by improving the overall gate dielectric constant. Finally, the on/off ratio strongly depends on the ribbon width. Compared to wider GNR devices with similar channel lengths, sub-10 nm GNRs can exhibit greatly increased on/off ratio. Therefore, the on/off ratio can be further increased by using smaller mask nanowires to create smaller bilayer GNRs. Our study demonstrates that the lateral confinement and vertical inversion symmetry breaking can work synergistically in bilayer GNRs to open up an effective pathway to graphene devices with sufficient on/off ratio for logic applications, and can impact significantly in areas including digital electronics, nanophotonics, and terahertz technology.

\section{Methods}

Fabrication and characterization of the Bilayer GNR devices. The silicon nanowires used here were grown by the Au nanocluster mediated vapour-liquid-solid growth approach, with the diamerters typically in the range of 30-60 nm and lengths of around $10 \mu \mathrm{m} . \mathrm{Al}_{2} \mathrm{O}_{3}$ outer shell was coated through atomic layer deposition (ALD) on the $\mathrm{Si}$ nanowire surface. $\mathrm{Si} / \mathrm{Al}_{2} \mathrm{O}_{3}$ core/shell nanowires were transferred by simply attaching nanowire grown wafer to target wafer. Nanowires were transferred onto target wafer surface by van der Waals force between the nanowires and the graphene surface. The fabrication method of bilayer GNR FETs is described in the main text. The d.c. electrical transport studies were conducted with a probe station at room temperature $\left(25^{\circ} \mathrm{C}\right)$ under ambient conditions with a computer-controlled analogue-to-digital converter. SEM imaging was performed on a JEOL 6700F unit operated at $5 \mathrm{kV}$.

1. Novoselov, K. S. et al. Electric field effect in atomically thin carbon films. Science 306, 666-669 (2004).

2. Novoselov, K. S. et al. Two-dimensional gas of massless Dirac fermions in graphene. Nature 438, 197-200 (2005).

3. Dean, C. R. et al. Boron nitride substrates for high-quality graphene electronics. Nat. Nanotechnol 5, 722-726 (2010).

4. Wu, Y. Q. et al. High-frequency, scaled graphene transistors on diamond-like carbon. Nature 472, 74-78 (2011).

5. Liao, L. et al. High-speed graphene transistors with a self-aligned nanowire gate. Nature 467, 305-308 (2010).

6. Wang, F. et al. Gate-variable optical transitions in graphene. Science 320, 206-209 (2008).

7. Xia, F., Mueller, T., Lin, Y.-M., Valdes-Garcia, A. \& Avouris, Ph. Ultrafast graphene photodetector. Nat. Nanotechnol. 4, 839-843 (2009).

8. Han, M. Y., Brant, J. C. \& Kim, P. Electron transport in disordered graphene nanoribbons. Phys. Rev. Lett. 104, 056801 (2010).

9. Gunlycke, D., Areshkin, D. A. \& White, C. T. Semiconducting graphene nanostrips with edge disorder. Appl. Phys. Lett. 90, 142104 (2007).

10. Lherbier, A. et al. Transport length scales in disordered graphene-based materials: strong localization regimes and dimensionality effects. Phys. Rev. Lett. 100, 036803 (2008).

11. Evaldsson, M. et al. Edge-disorder-induced Anderson localization and conduction gap in graphene nanoribbons. Phys. Rev. B 78, 161407(R) (2008).

12. Querlioz, D. et al. Suppression of the orientation effects on bandgap in graphene nanoribbons in the presence of edge disorder. Appl. Phys. Lett. 92, 042108 (2008).

13. Mucciolo, E. R., Castro Neto, A. H. \& Lewenkopf, C. H. Conductance quantization and transport gaps in disordered graphene nanoribbons. Phys. Rev. B 79, 075407 (2009).

14. Martin, I. \& Blanter, Y. M. Transport in disordered graphene nanoribbons. Phys. Rev. B 79, 235132 (2009).

15. Han, M. Y., Ozyilmaz, B., Zhang, Y. \& Kim, P. Energy band-gap engineering of graphene nanoribbons. Phys. Rev. Lett. 98, 206805 (2007).

16. Li, X., Wang, X., Zhang, L., Lee, S. \& Dai, H. Chemically derived, ultrasmooth graphene nanoribbon semiconductors. Science 319, 1229-1232 (2008).

17. Bai, J., Duan, X. \& Huang, Y. Rational fabrication of graphene nanoribbons using a nanowire etch mask. Nano Lett. 9, 2083-2087 (2009).

18. Wang, X. \& Dai, H. Etching and narrowing of graphene from the edges. Nat. Chem. 2, 661-665 (2010). 
19. Bai, J., Zhong, X., Jiang, S., Huang, Y. \& Duan, X. Graphene nanomesh. Nat. Nanotechnol. 5, 190-194 (2010).

20. Ponomarenko, L. A. et al. Chaotic dirac billiard in graphene quantum dots. Science 320, 356 (2008).

21. Zhang, Y. et al. Direct observation of a widely tunable bandgap in bilayer graphene. Nature 459, 820-823 (2009).

22. Castro, E. V. et al. Biased bilayer graphene: semiconductor with a gap tunable by the electric field effect. Phys. Rev. Lett. 99, 216802 (2007).

23. Ohta, T., Bostwick, A., Seyller, Th., Horn, K. \& Rotenberg, E. Controlling the electronic structure of bilayer graphene. Science 313, 951-954 (2006).

24. Xia, F., Farmer, D. B., Lin, Y. \& Avouris, Ph. Graphene field-effect transistors with high on/off current ratio and large transport bandgap at room temperature. Nano Lett. 10, 715-718 (2010).

25. Yu, W. J., Liao, L., Chae, S. H., Lee, Y. H. \& Duan, X. Toward tunable bandgap and tunable dirac point in bilayer graphene with molecular doping. Nano Lett. 11, 4759-4763 (2011).

26. Sahu, B., Min, H., MacDonald, A. H. \& Banerjee, S. K. Energy gaps, magnetism, and electric-field effects in bilayer graphene nanoribbons. Phys. Rev. B 78, 045404 (2008).

27. Lima, M. P., Fazzio, A. \& Silva, A. J. R. Edge effects in bilayer graphene nanoribbons: Ab initio total-energy density functional theory calculations. Phys. Rev. B 79, 153401 (2009).

28. Kumar, B. \& Guo, J. Multilayer graphene nanoribbon under vertical electric field. J. Appl. Phys. 110, 044309 (2011).

29. Park, S. \& Ruoff, R. S. Chemical methods for the production of graphenes. Nat. Nanotechnol. 4, 217-224 (2009).

30. Tung, V. C., Allen, M. J., Yang, Y. \& Kaner, R. B. High-throughput solution processing of large-scale graphene. Nat. Nanotechol. 4, 25-29 (2009).

31. Li, X. et al. Large-area synthesis of high quality and uniform graphene films on copper foils. Science 324, 1312-1314 (2009).

32. Reina, A. et al. Large area, few-layer graphene films on arbitrary substrates by chemical vapour deposition. Nano Lett. 9, 30-35 (2009).

33. Blake, P. et al. Making graphene visible. Appl. Phys. Lett. 91, 063124 (2007).

34. Ferrari, A. C. et al. Raman spectrum of graphene and graphene layers. Phy. Rev. Lett. 97, 187401 (2006)
35. Liao, L. et al. High-kappa oxide nanoribbons as gate dielectrics for high mobility top-gated graphene transistors. Proc. Natl. Acad. Sci. USA 107, 6711-6715 (2010).

36. Liao, L. et al. Top-gated graphene nanoribbon transistors with ultrathin high-k dielectrics. Nano Lett. 10, 1917-1921 (2010).

37. Yang, R., Shi, Z., Zhang, L., Shi, D. \& Zhang, G. Observation of raman G-peak split for graphene nanoribbons with hydrogen-terminated zigzag edges. Nano Lett. 11, 4083-4088 (2011).

38. Schwierz, F. Graphene transistors. Nat. Nanotechnol. 5, 487-496 (2010).

39. Bai, J. et al. Very large magnetoresistance in graphene nanoribbons. Nat. Nanotechnol. 5, 655-659 (2010).

\section{Acknowledgements}

We acknowledge the Nanoelectronics Research Facility (NRF) at UCLA for technical support. X.D. acknowledges financial support by NSF CAREER award 0956171. W.J.Y. acknowledges partial support by the National Research Foundation of Korea Grant funded by the Korean Government (Ministry of Education, Science and Technology) [NRF-2011-351-c00034]

\section{Author contributions}

X.D. and W.J.Y. conceived and designed the experiments. W.J.Y. performed the experiments. X.D. and W.J.Y. analysed the data and co-wrote the paper.

\section{Additional information}

Supplementary information accompanies this paper at http://www.nature.com/ scientificreports

Competing financial interests: The authors declare no competing financial interests.

License: This work is licensed under a Creative Commons

Attribution-NonCommercial-NoDerivs 3.0 Unported License. To view a copy of this license, visit http://creativecommons.org/licenses/by-nc-nd/3.0/

How to cite this article: Yu, W.J. \& Duan, X.F. Tunable transport gap in narrow bilayer graphene nanoribbons. Sci. Rep. 3, 1248; DOI:10.1038/srep01248 (2013). 